\title{
Engineering NIR-IIb fluorescence of Er-based lanthanide nanoparticles for through-skull targeted imaging and imaging-guided surgery of orthotopic glioma
}

\author{
Feng Ren ${ }^{\mathrm{a}}$, Hanghang Liu ${ }^{\mathrm{a}}$, Hao Zhang ${ }^{\mathrm{a}}$, Zhilin Jiang ${ }^{\mathrm{a}}$, Bing Xia ${ }^{\mathrm{b}}$, Cécile Genevois ${ }^{\mathrm{c}}$, \\ Tao He ${ }^{\mathrm{b}}$, Mathieu Allix ${ }^{\mathrm{c}}$, Qiao Sun ${ }^{\mathrm{a}}$, Zhen $\mathrm{Li}^{\mathrm{a}, *}$, Mingyuan Gao ${ }^{\mathrm{a}}$ \\ a Center for Molecular Imaging and Nuclear Medicine, State Key Laboratory of Radiation Medicine and Protection, School for Radiological and \\ Interdisciplinary Sciences (RAD-X), Soochow University, Collaborative Innovation Center of Radiation Medicine of Jiangsu Higher Education Institutions, \\ Suzhou, 215123, PR China \\ b School of Chemistry and Chemical Engineering, Anhui Province Key Laboratory of Advanced Catalytic Materials and Reaction Engineering, Hefei \\ University of Technology, Hefei, Anhui, 230009, PR China \\ ' CNRS, CEMHTI UPR3079, Université Orléans, F-45071, Orléans, France
}

Keywords:

Er-based lanthanide nanoparticles NIR IIb fluorescence imaging

Imaging-guided surgery

Focused ultrasound sonication

Orthotopic glioma

\begin{abstract}
A B S T R A C T
Highly sensitive and specific discrimination of brain tumor margins from the surrounding parenchyma remains a formidable challenge. Limited by the short of photostable probes with deep tissue penetration and high efficiency of crossing the blood-brain-barrier (BBB), the development of fluorescence-guided surgery (FGS) of brain tumors was markedly constrained. Herein, we report the capability of the strong second near-infrared-IIb (NIR IIb, 1500-1700 nm) fluorescence from Er-based lanthanide nanoparticles in imaging-guided surgery of orthotopic glioma. We designed an energy-cascaded $\mathrm{Er}^{3+}-\mathrm{Ce}^{3+}-\mathrm{A}^{3+}(\mathrm{A}$ $=\mathrm{Yb}, \mathrm{Ho}, \mathrm{Tm}$ ) system and prepared a series of $\mathrm{NaErF}_{4}: \mathrm{Ce}_{\mathrm{N} a A F_{4}} @ \mathrm{NaLuF}_{4}$ down-conversion nanoparticles (DCNPs) for optimizing the influence of $\mathrm{NaAF}_{4}$ interlayer and $\mathrm{Ce}^{3+}$ dopants. We modified the optimal NaErF $4: 2.5 \% C e @ \mathrm{NaYbF}_{4}(0.9 \mathrm{~nm}) @ \mathrm{NaLuF}_{4}$ DCNPs with Dye-brush polymer (Dye-BP) to facilitate ${ }^{4} \mathrm{I}_{13 / 2} \rightarrow{ }^{4} \mathrm{I}_{15 / 2}$ transition, which leads to an impressive 675-fold enhancement of $1525 \mathrm{~nm}$ fluorescence in aqueous solution under $808 \mathrm{~nm}$ excitation due to the excellent energy-cascaded downconversion (ECD), in comparison with that of $\mathrm{NaErF}_{4}$ nanoparticles. We further modified these highly bright nanoparticles with tumor-targeting angiopep-2 peptide, and efficiently delivered them to the glioma by using the focused ultrasound sonication (FUS) to temporarily open the BBB. We obtained the highest tumorto-background ratio ( $\mathrm{TBR}=12.5$ ) ever reported in the targeted NIR IIb fluorescence imaging of small orthotopic glioma (size $<3 \mathrm{~mm}$, depth> $3 \mathrm{~mm}$ ) through intact skull and scalp, which was drastically improved to $\sim 150$ after cardiac perfusion and craniotomy to ensure the precise resection of tumor. More importantly, the size of glioma measured from the width of fluorescence profile is very close to that from $T_{2}$-weighted MRI images. Our work provides the insights into engineering NIR IIb fluorescence of lanthanide nanoparticles, and demonstrates the great potential of NIR IIb fluorescence imaging-guided surgery of tumor.
\end{abstract}

\section{Introduction}

Finely and clearly visualizing the margins of brain tumors, especially for glioblastoma (GBM), which is the most common malignant brain tumor, from the surrounding parenchyma is the lynchpin for their precise diagnosis and surgery [1-3]. Although the typical use of visual inspection and imaging guidance can pro- vide valuable information for clinicians, the intrinsic limitations of currently available imaging methods, such as low sensitivity, non-dynamical inspection, and hazardous ionizing radiation, can cause intraoperative failure in completely resecting tumor tissues [1,4-6]. In addition, for fluorescence-guided surgery (FGS) of brain tumors, photostable probes with strong capability of crossing the blood-brain-barrier (BBB) are crucial for precise delineation glioma margin and the subsequent curative resection. The intrinsic drawbacks of clinically used probes (5-aminolevulinic acid (5-ALA) [7,8] and indocyanine green (ICG) $[9,10])$, such as insufficient photostability [11] and short excitation/emission wavelength-induced low 
tissue penetration depth, are driving the development of alternative probes for FGS of brain tumors.

Recently, much effort has been devoted to developing the second near-infrared (NIR II, 1000-1700 nm) fluorescence imaging favoring for reduced tissue absorption, scattering and minimized auto-fluorescence $[12,13]$. In particular, the fluorescence in the range of $1500-1700 \mathrm{~nm}$ (NIR IIb) has attracted considerable interests, because it offers the lowest photon scattering effect, according to the Mie theory $\left(\mu_{s}{ }^{\prime} \propto \lambda^{-\alpha}, \mu_{s}\right.$ ' represents the reduced scattering coefficient, $\lambda$ is the wavelength of incident light, $\alpha=0.2-4$ for different tissues) [14], and the negligible autofluorescence under laser irradiation in this window $[15,16]$. Currently, the NIR IIb fluorescent probes, such as single-walled carbon nanotubes [16], PbS@CdS quantum dots [17], organic dyes [18], have been applied for imaging mouse brain vasculature through intact skull and scalp. Despite of their advances, some other limitations drive the research of complementary nanoprobes $[19,20]$. Alternatively, Er-based rareearth nanoparticles are well-known for up-conversion (UC) and down-conversion (DC) fluorescence from visible to NIR II region with controllable size, shape and functionality [21-24]. Typical $\mathrm{Yb}^{3+}-\mathrm{Er}^{3+}$ co-doped lanthanide nanoparticles could emit NIR IIb fluorescence under $980 \mathrm{~nm}$ light excitation, but the water in tissues would inevitably attenuate $980 \mathrm{~nm}$ light to produce local heat and reduce penetration depth [25]. Although introducing $\mathrm{Nd}^{3+}$ sensitizers into $\mathrm{Yb}^{3+}-\mathrm{Er}^{3+}$ co-doped nanoparticles makes the feasibility of excitation with $\sim 800 \mathrm{~nm}$ light [26], precise control of core and shell is demanded for optimizing NIR IIb emission.

Unlike other lanthanide ions serving as either sensitizers or activators, $\mathrm{Er}^{3+}$ ions can act as both the sensitizers and activators to render effective UC and DC processes [22]. To increase near-infrared absorption as much as possible, high concentration of sensitizers is usually required [27], which can inevitably cause non-radiative cross-relaxation and energy migration loss of $\mathrm{Er}^{3+}$-heavily doped nanoparticles. To reduce the negative effects caused by activators $\left(\mathrm{Er}^{3+}\right.$ ions), various energy trapping centers $\left(\mathrm{Yb}^{3+}, \mathrm{Tm}^{3+}, \mathrm{Ho}^{3+}\right.$, etc) were introduced to confine the excitation energy to enhance the UC process $[28,29]$. However, there is rare report on boosting DC process of $\mathrm{Er}^{3+}$-heavily doped lanthanide nanoparticles, which are facing the challenge of weak NIR IIb fluorescence.

To significantly improve NIR IIb emission of $\mathrm{Er}^{3+}$-heavily doped nanoparticles under $808 \mathrm{~nm}$ excitation, maximally increasing absorption and optimizing the energy transfer to ${ }^{4} \mathrm{I}_{13 / 2}$ are crucial. Herein, we report an energy-cascaded strategy to boost NIR IIb emission of Er-based core-shell-shell down-conversion nanoparticles (DCNPs), and a cooperative tactic to deliver them to orthotopic glioma for targeted imaging and imaging-guided surgery. The resulting nanoparticles exhibit 675-fold enhancement of emission at $1525 \mathrm{~nm}$ in aqueous solution in comparison with core nanoparticles. Their strong NIR IIb fluorescence and low background make them very useful in imaging and imaging-guided surgery of deepseated tumor.

\section{Results and discussion}

\section{Modulating NIR IIb emission of Er-based DCNPs via energy-cascaded process}

We designed core-shell-shell $\mathrm{NaErF}_{4}: \mathrm{Ce} @ \mathrm{NaYbF}_{4} @ \mathrm{NaLuF}_{4}$ DCNPs by using excitation energy trapping and non-radiative cross-relaxation approaches (Fig. 1a) $[20,29,30]$. In this energycascaded $\mathrm{Er}^{3+}-\mathrm{Ce}^{3+}-\mathrm{Yb}^{3+}$ system, $\mathrm{NaYbF}_{4}$ interlayer can bounce back the radiative energy from ${ }^{4} \mathrm{I}_{11 / 2}$ of $\mathrm{Er}^{3+}$, and sequentially drive cross-relaxation to ${ }^{4} \mathrm{I}_{13 / 2}$ of $\mathrm{Er}^{3+}$ via $\mathrm{Ce}^{3+}$ ions to maximize ${ }^{4} \mathrm{I}_{13 / 2} \rightarrow{ }^{4} \mathrm{I}_{15 / 2}$ transition (Fig. 1b). Although other self-sensitized activators, such as $\mathrm{Er}^{3+}, \mathrm{Ho}^{3+}$, and $\mathrm{Tm}^{3+}$ ions, could promote the energy transfer from ${ }^{4} \mathrm{I}_{11 / 2}$ to ${ }^{4} \mathrm{I}_{13 / 2}$ of $\mathrm{Er}^{3+}$ with two involved energy levels (Fig. 1c), we hypothesize that $\mathrm{Er}^{3+}-\mathrm{Ce}^{3+}-\mathrm{Yb}^{3+}$ system is more favorable for ${ }^{4} \mathrm{I}_{13 / 2} \rightarrow{ }^{4} \mathrm{I}_{15 / 2}$ transition to generate NIR IIb fluorescence, due to the presence of radiative photon relaxation induced by the intermediate energy level (dash line in Fig. 1c).

We prepared a series of $\mathrm{NaErF}_{4} @ \mathrm{NaAF}_{4}(\mathrm{~A}=\mathrm{Yb}, \mathrm{Ho}, \mathrm{Tm}) @ \mathrm{NaLuF}_{4}$ nanoparticles with varied interlayer thickness by simply changing the amount of cluster precursors. The inert $\mathrm{NaLuF}_{4}$ shell was used to inhibit surface quenching effect of Er-based nanoparticles [22,31]. Transmission electron microscopy (TEM) images of these nanoparticles are shown in Fig. S1-3, the effects of their interlayer thickness on UC (655 nm emission) and DC (1525 nm emission) processes are also distinctively illustrated in Fig. $1 \mathrm{~d}$. When $\mathrm{NaYbF}_{4}$ was used as the interlayer, the optimized $\mathrm{NaYbF}_{4}(0.9 \mathrm{~nm}$ in thickness) endowed 1.8-fold and 5.8-fold enhancement of UC (655 nm) and DC (1525 nm) emissions compared to those of $\mathrm{NaErF}_{4} @ \mathrm{NaLuF}_{4}$ nanoparticles (Figs. $1 \mathrm{~d}$ and $\mathrm{S} 4 \mathrm{a}$ ). For $\mathrm{NaHoF}_{4}$ interlayer, the optimized $\mathrm{NaHoF}_{4}(0.2 \mathrm{~nm}$ in thickness) promoted the UC process to result in 4.8-fold enhancement of $655 \mathrm{~nm}$ emission, which is nearly twice of $1525 \mathrm{~nm}$ emission (Figs. $1 \mathrm{~d}$ and S4b). The dominant UC process could be attributed to that $\mathrm{Ho}^{3+}$ ions with ${ }^{5} \mathrm{I}_{6}$ level confined excitation energy to ${ }^{4} \mathrm{I}_{13 / 2}$ of $\mathrm{Er}^{3+}$ and immediately integrated 808 $\mathrm{nm}$ photons to generate up-conversion emission (Fig. 1c). Since $\mathrm{Tm}^{3+}$ ions have serious problem with cross-relaxation [32], the $\mathrm{NaTmF}_{4}$ interlayer has no contribution to UC and DC processes with the variation of thickness from 0.4 to $2.1 \mathrm{~nm}$ (Figs. 1d and S4c). The above results demonstrate that $\mathrm{Yb}^{3+}$ and $\mathrm{Ho}^{3+}$ ions can efficiently trap excitation energy to simultaneously promote UC and DC processes, and show the limited improvement of ${ }^{4} \mathrm{I}_{13 / 2} \rightarrow{ }^{4} \mathrm{I}_{15 / 2}$ transition to generate NIR IIb emission.

Recent progress has exemplified that $\mathrm{Ce}^{3+}$ ions can efficiently shorten the ${ }^{4} \mathrm{I}_{11 / 2}$ of $\mathrm{Er}^{3+}$ lifetime to enhance the DC process and improve emission beyond $1500 \mathrm{~nm}$ under irradiation by 980 nm light $[19,20,33]$. But for the $\mathrm{Er}^{3+}$-heavily doped nanoparticles excited with $808 \mathrm{~nm}$ light, the effect and the role of $\mathrm{Ce}^{3+}$ ions are still unknown. We firstly tested the effect of $\mathrm{Ce}^{3+}$ dopant on the ${ }^{4} \mathrm{I}_{13 / 2} \rightarrow{ }^{4} \mathrm{I}_{15 / 2}$ transition. TEM images of prepared $\mathrm{NaErF}_{4}: \mathrm{y} \% \mathrm{Ce}$ (y $=0,1,2.5,5) @ \mathrm{NaLuF}_{4}$ nanoparticles in Fig. S5a-d show an average size of $12.5 \mathrm{~nm}$ with $1.1 \mathrm{~nm}$ deviation (Fig. S5e). The optimized doping ratio of $\mathrm{Ce}^{3+}$ ions (2.5\%) leads to1.4-fold enhancement of 1525 $\mathrm{nm}$ fluorescence at the cost of 2.9-fold decreases of $655 \mathrm{~nm}$ fluorescence (Fig. S5f), which proves the effectiveness of $\mathrm{Ce}^{3+}$ dopants.

Based on the above results, we introduced $\mathrm{Ce}^{3+}$ dopants into $\mathrm{NaErF}_{4} @ \mathrm{NaYbF}_{4}(0.9 \mathrm{~nm}) @ \mathrm{NaLuF}_{4}$ nanoparticles because the $\mathrm{Yb}^{3+}$ ions can trap energy to ${ }^{4} \mathrm{I}_{11 / 2}$ of $\mathrm{Er}^{3+}$ more efficiently than other ions $\left(\mathrm{Er}^{3+}, \mathrm{Ho}^{3+}, \mathrm{Tm}^{3+}\right)$, and cooperate with $\mathrm{Ce}^{3+}$ dopants to boost ${ }^{4} \mathrm{I}_{13 / 2} \rightarrow{ }^{4} \mathrm{I}_{15 / 2}$ transition by populating the ${ }^{4} \mathrm{I}_{13 / 2}$ of $\mathrm{Er}^{3+}$. Uniform size and spherical morphology of $\mathrm{NaErF}_{4}: \mathrm{y} \% \mathrm{Ce}(\mathrm{y}=1$, 2.5, 5, 10)@NaYbF $4(0.9 \mathrm{~nm}) @ \mathrm{NaLuF}_{4}$ nanoparticles were prepared and characterized by TEM (Fig. S6). Their corresponding fluorescence spectra present that the optimized doping concentration (2.5\%) leads to 3.5-fold enhancement of $1525 \mathrm{~nm}$ fluorescence(Fig. S7a). More importantly, the optimized $\mathrm{NaErF}_{4}: 2.5$ \%Ce@ $\mathrm{NaYbF}_{4}(0.9 \mathrm{~nm}) @ \mathrm{NaLuF}_{4}$ nanoparticles show an impressive enhancement of $1525 \mathrm{~nm}$ fluorescence (13.4-folds), compared to that of $\mathrm{NaErF}_{4} @ \mathrm{NaLuF}_{4}$ nanoparticles under the excitation of 808 $\mathrm{nm}$ light (Fig. 1d). In addition, the optimized $\mathrm{Ce}^{3+}$ dopants can also drastically increase the lifetime of down-conversion luminescence at $1525 \mathrm{~nm}$ to $2.88 \mathrm{~ms}$, which is much higher than that of $\mathrm{NaErF}_{4} @ \mathrm{NaLuF}_{4}$ nanoparticles $(0.28 \mathrm{~ms})$ and $\mathrm{NaErF}_{4} @ \mathrm{NaYbF}_{4}(0.9$ $\mathrm{nm}) @ \mathrm{NaLuF}_{4}$ nanoparticles $(0.4 \mathrm{~ms})$ (Fig. S7b-c). The results demonstrate that $\mathrm{Ce}^{3+}$ dopants induced population of ${ }^{4} \mathrm{I}_{13 / 2}$ energy level of $\mathrm{Er}^{3+}$ ions, which can enhance the NIR IIb fluorescence and prolong its lifetime simultaneously.

A representative TEM image of the optimized $\mathrm{NaErF}_{4}: 2.5$ \%Ce@NaYbF 4 (0.9 nm)@NaLuF 4 nanoparticles (Er-DCNPs) shows 


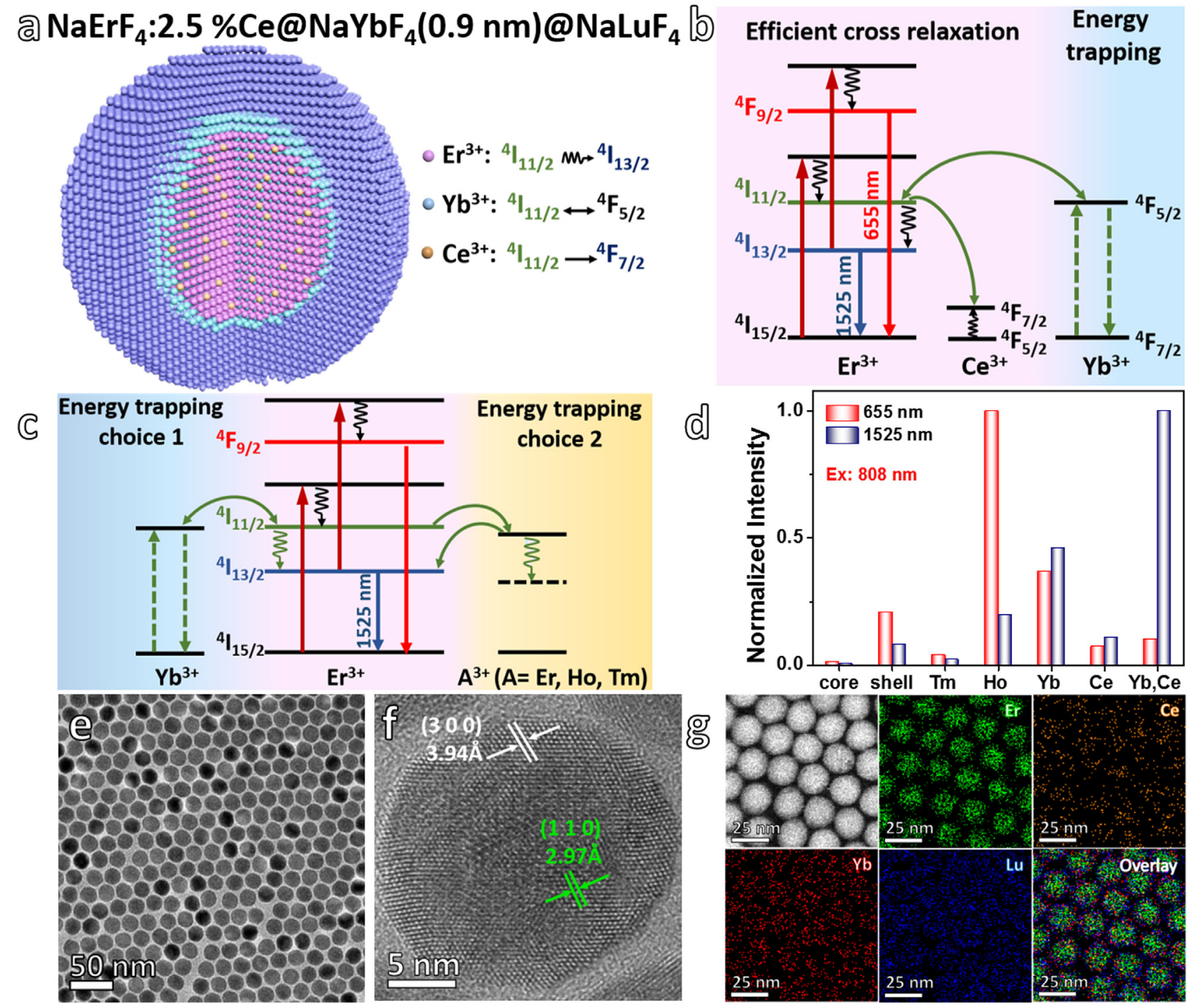

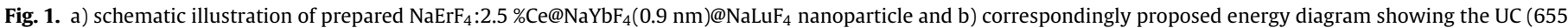

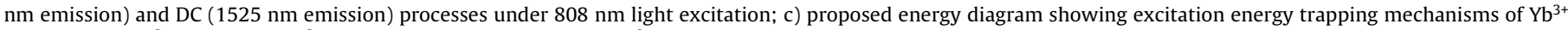

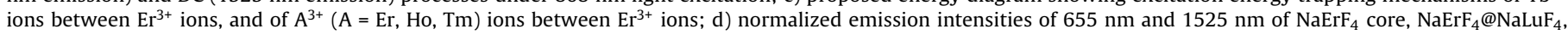

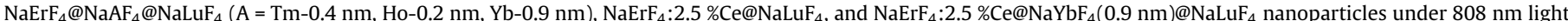

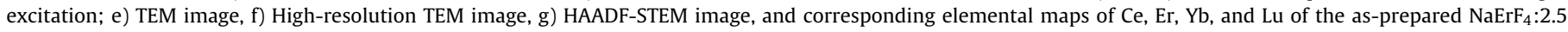
\%Ce@NaYbF 4 (0.9 nm)@NaLuF 4 nanoparticles.

highly uniform and monodispersed spherical particles with a diameter of $17.9 \pm 0.9 \mathrm{~nm}$ (Fig. 1e). Their core-shell structure and high crystallinity are demonstrated by their corresponding highresolution TEM image (HRTEM, Fig. 1f). The lattice spacings for the core and shell are measured to be $\sim 2.97 \AA$ and $\sim 3.94 \AA$, respectively, matching well with the (110) planes of hexagonal-phase $\mathrm{NaErF}_{4}$ (PDF\#27-0689) and the (300) planes of hexagonal-phase $\mathrm{NaLuF}_{4}$ (PDF\#48-0830). The high-angle annular dark field-scanning TEM (HAADF-STEM) and corresponding elemental maps of Er-DCNPs exhibit homogeneous distribution of $\mathrm{Er}^{3+}$ in the core of the NPs, along with successful doping of $\mathrm{Ce}^{3+}$ ions and $\mathrm{Yb}^{3+} / \mathrm{Lu}^{3+}$ ions localized in the shells (Fig. 1g).

Surface modification of Er-DCNPs for further enhancement of their NIR IIb fluorescence and biocompatibility

For in vivo bio-imaging, a hydrophilic surface is required to endow biocompatibility to Er-DCNPs. Due to the quenching effect of hydroxyl groups on the DC emission $(\sim 1500 \mathrm{~nm})$ and the nar- row ( $\sim 10 \mathrm{~nm}$ ) and weak absorption (cross-section of $10^{-20} \mathrm{~cm}^{-2}$ ) of $\mathrm{Er}^{3+}$ ions [20,27], amphiphilic polymers such as DSPE-PEG were usually used to wrap dyes (e.g. IR-808, IR-806) as organic sensitizer, which improved the solubility of nanoparticles and the NIR II fluorescence [34-36]. However, the wrapped dyes could be leaked and instable, which directly influenced the sensitizing efficiency in vitro and in vivo. To resolve this issue, we designed and synthesized a novel dye-brush polymer (Dye-BP) through reversible addition-fragmentation chain transfer (RAFT) polymerization to integrate surface ligands with dye sensitizer (see the details on the synthesis and characterization of Dye-BP in the Supporting Information, Schemes S1-2, and Figs. S8-14). Following the same synthetic route, we also prepared a similar brush polymer (BP) without dye. By using different ratios of BP and Dye-BP, we can tune the amount of dye on the surface of nanoparticles to achieve the optimized sensitization effect, and simultaneously provide biocompatibility and functional groups for conjugating with targeting molecules (Fig. 2a). Fig. 2b shows the proposed energycascaded down-conversion (ECD) process, where the dye as the 


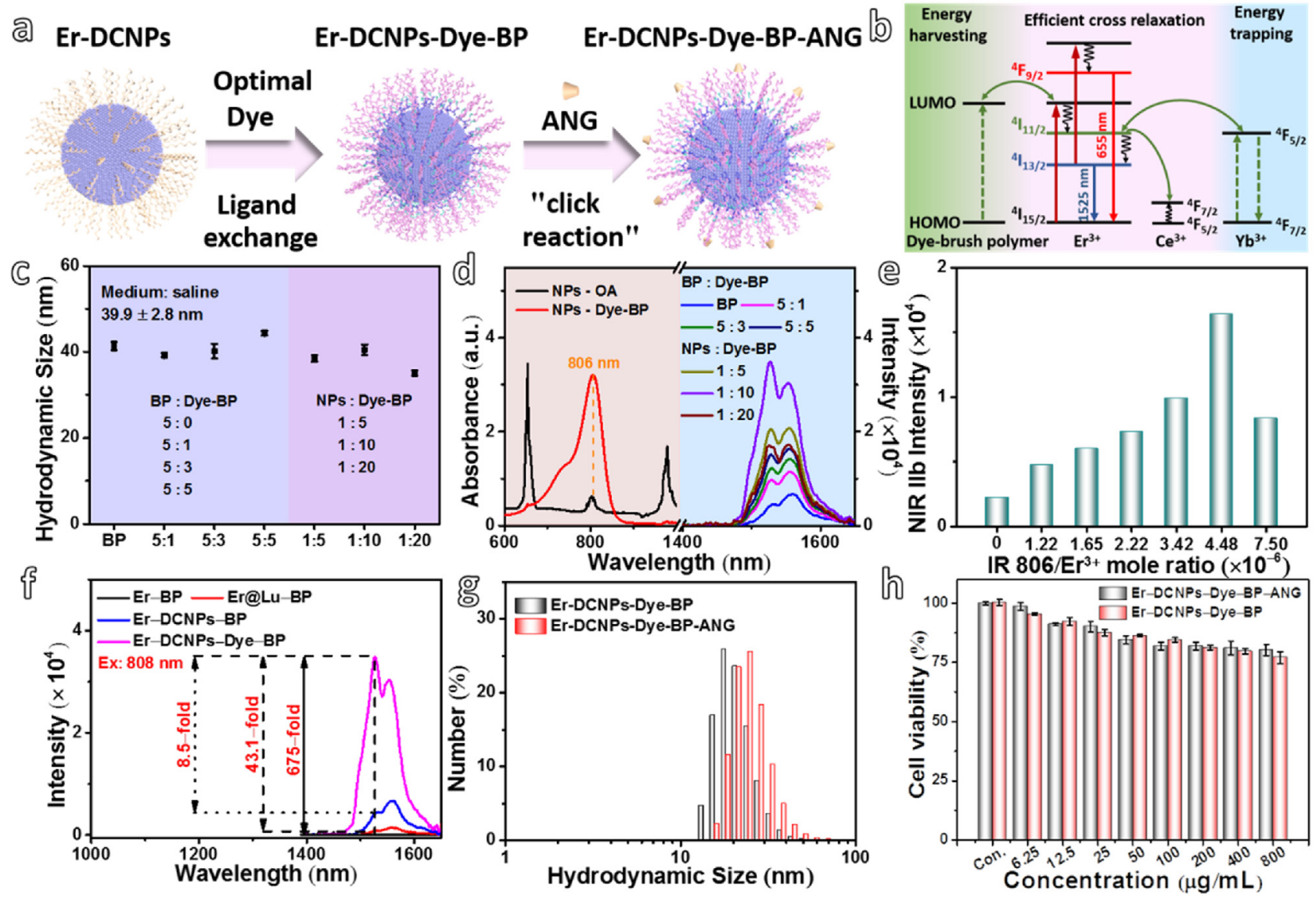

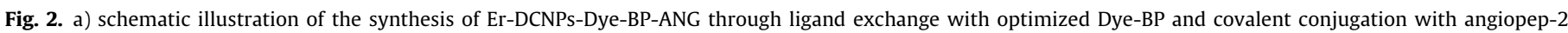

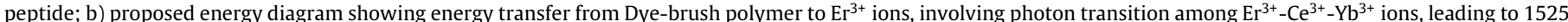

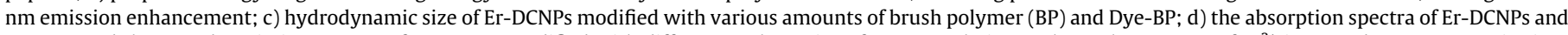

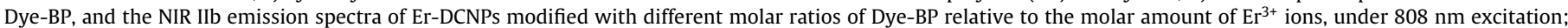

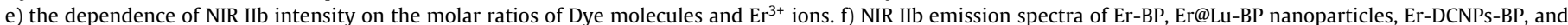

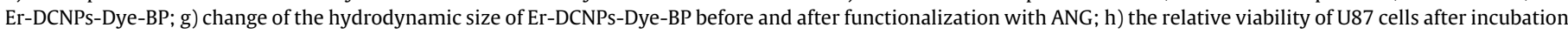
with Er-DCNPs-Dye-BP and Er-DCNPs-Dye-BP-ANG for $24 \mathrm{~h}$.

excitation-energy co-harvester absorb $808 \mathrm{~nm}$ photons and transfer to ${ }^{4} \mathrm{I}_{9 / 2}$ of $\mathrm{Er}^{3+}$, and then activate the energy-trapping $\mathrm{Yb}^{3+}$ ions and cross-relaxation $\mathrm{Ce}^{3+}$ ions to boost ${ }^{4} \mathrm{I}_{13 / 2} \rightarrow{ }^{4} \mathrm{I}_{15 / 2}$ transition in the Er-DCNPs.

The Er-DCNPs modified with varied mole ratios of BP to Dye-BP exhibit a similar average hydrodynamic size (39.9 nm) with a very small deviation $(2.8 \mathrm{~nm})$ (Fig. 2c), which indicates superior water-dispersity of polymer-modified Er-DCNPs. The highly overlapping absorption of Dye-BP and Er-DCNPs at $808 \mathrm{~nm}$ (Fig. 2d-left) laid a solid ground for maximizing the ECD process. The NIR IIb fluorescence $(1525 \mathrm{~nm})$ of resultant Er-DCNPs-Dye-BP was enhanced by 8.5 -fold in comparison with that of Er-DCNPs-BP (Fig. 2d-right), and the mole ratio of dye to $\mathrm{Er}^{3+}$ ions was calculated to be $4.48 \times 10^{-6}$ based on UV absorption quantitation (Figs. 2e and S15). In addition, the NIR IIb emission of optimized Dye-BP modified ErDCNPs (Er-DCNPs-Dye-BP) in aqueous solution was impressively improved by 43.1-fold and 675-fold compared to that of BP-coated $\mathrm{NaErF}_{4} @ \mathrm{NaLuF}_{4}$ (Er@Lu-BP) and $\mathrm{NaErF}_{4}$ (Er-BP) nanoparticles, respectively (Fig. $2 \mathrm{f}$ ).

Their fourier transform infrared (FTIR) spectra confirm the successful surface modification of Er-DCNPs with Dye-BP (Fig. S16a). TEM image of Er-DCNPs-Dye-BP shows excellent dispersibility, with a similar size to that of oleic acid capped Er-DCNPs (i.e. $18 \mathrm{~nm}$, Fig. S16b-c). The strong coordination between the phosphate groups of brush polymer and the surface lanthanide ions of nanoparticles guarantees the excellent colloidal stability of the nanoparticles in $0.9 \mathrm{wt} \% \mathrm{NaCl}$ solution [37], as evidenced by the similar hydrodynamic size around $34 \mathrm{~nm}$ retained over one week of storage (Fig. S16d-e).

\section{Targeting capability of Er-DCNPs-Dye-BP-ANG}

To enhance the uptake by glioma cells, we functionalized Er-DCNPs-Dye-BP with angiopep-2 peptide (ANG), which can specifically bind to overexpressed low density lipoprotein receptor-related protein (LRP) on glioma cells [38-40]. The increment in the hydrodynamic size of the nanoparticles after modification was measured to be $6.2 \mathrm{~nm}$ (Fig. $2 \mathrm{~g}$ ). The in vitro cytotoxicity of the nanoparticles against glioma cells was tested by a typical MTT assay (Fig. 2h). The viability of glioma cells is over $75 \%$, even though they were incubated with a high concentration of nanoparticles $(800 \mu \mathrm{g} / \mathrm{mL})$ for $24 \mathrm{~h}$, which demonstrates the excellent biocompatibility of Er-DCNPs-Dye-BP modified with or without functional angiopep-2 peptide. Since our nanoparticles have both UC and DC luminescence, we used the UC luminescence to characterize their uptake by glioma cells (Figs. 3a and S16f). The in vitro confocal microscope images in Fig. 3b show that strong UC fluorescence under excitation by the $980 \mathrm{~nm}$ laser was observed around the nuclei of the glioma cells after incubation with Er-DCNPs-Dye-BP-ANG, implying that they were efficiently endocytosed by glioma cells into cytoplasm. In contrast, 

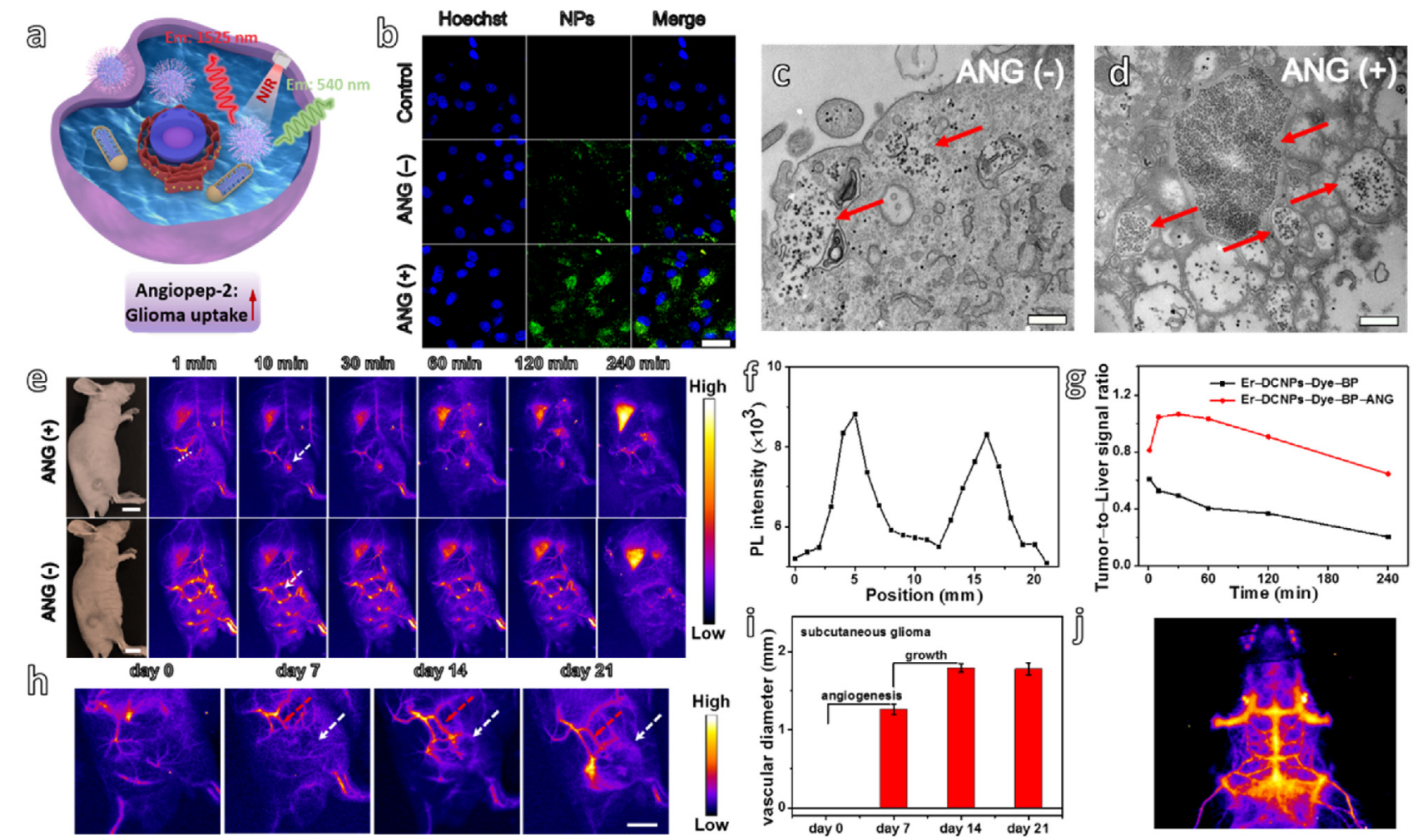

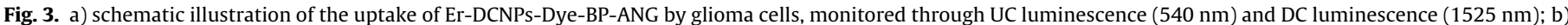

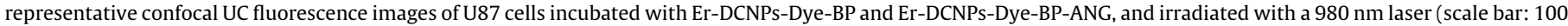

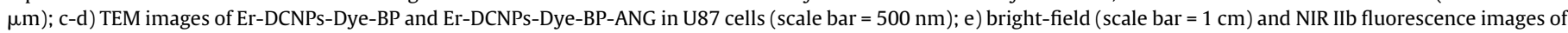

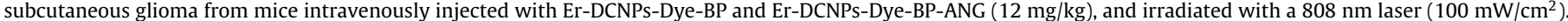

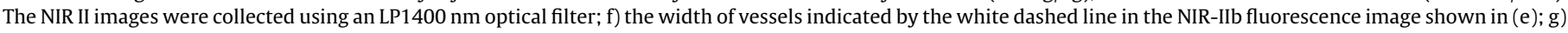

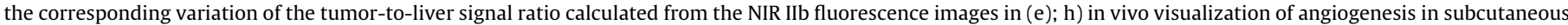

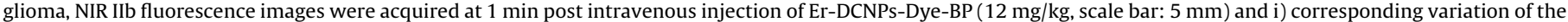
vascular width (red arrow pointed); j) NIR IIb fluorescence imaging of brain vasculature in a healthy nude mouse.

the cells incubated with Er-DCNPs-Dye-BP only show weak fluorescence. Additionally, the representative TEM images show more nanoparticles distributed in the cytoplasm of cells incubated with Er-DCNPs-Dye-BP-ANG, which further proves the high specificity of covalent peptide to glioma (Fig. $3 c-d$ ).

To assess the specificity of Er-DCNPs-Dye-BP-ANG in vivo, NIR IIb fluorescence imaging of subcutaneously grafted glioma tumors was carried out with intravenous injection of passive-targeting (ANG(-) group) and active targeting (ANG(+) group) nanoparticles $(12 \mathrm{mg} / \mathrm{kg}$ ) (Fig. 3e). The $808 \mathrm{~nm}$ laser with a power density of $100 \mathrm{~mW} / \mathrm{cm}^{2}$ was used for the NIR IIb imaging. The width of blood vessels could be measured by the fluorescence signal, e.g. two blood vessels (white dashed line in Fig. 3e) in the NIR IIb fluorescence image of a mouse acquired at $1 \mathrm{~min}$ post-injection of Er-DCNPs-Dye-BP-ANG were measured to be $6 \mathrm{~mm}$ and $7 \mathrm{~mm}$ (Fig. 3f), respectively. More importantly, the tumor from the ANG(+) group gradually became bright and achieved its maximal brightness after 30 min post-injection, which is much brighter than for the ANG(-) group. The calculated tumor-to-liver fluorescence ratio from the ANG $(+)$ group collected at 30 min post-injection is 2.2 -fold that from the ANG(-) group (Fig. $3 \mathrm{~g}$ ), due to the excellent targeting capability of ANG peptide to the LRP receptor [38]. Moreover, the superior NIR IIb fluorescence of well-designed Er-DCNPs-Dye-BP can be applied to monitor the evolution of tumor related vessels (labeled with red arrow in Fig. 3h), which clearly demonstrates the angiogenesis process in the subcutaneous glioma, evidenced by the variation of a typical vessel increased from 0 to $1.26 \mathrm{~mm}$ in the first week, then to $1.79 \mathrm{~mm}$ till day 14 , and maintained at $1.79 \mathrm{~mm}$ on day 21 (Fig. 3i).
As the basis for the targeted imaging of orthotopic glioma, NIR IIb fluorescence imaging of brain vasculature and circulation, distribution and toxicity of our well-designed nanoprobes (Er-DCNPs-Dye-BP) in healthy mice were conducted. The NIR IIb fluorescence image of a mouse brain was immediately collected under excitation by the $808 \mathrm{~nm}$ laser $\left(120 \mathrm{~mW} / \mathrm{cm}^{2}\right)$ after tail vein injection of nanoprobes $(20 \mathrm{mg} / \mathrm{kg}$ ) (Fig. $3 \mathrm{j}$ ). The brain vascular network was clearly observed due to the strong luminescence of our nanoprobes and the deep penetration of the NIR IIb fluorescence through the intact mouse's scalp and skull.

\section{Bio-circulation, distribution and toxicity of Er-DCNPs-Dye-BP}

Highly sensitive single-photon emission computed tomography (SPECT) imaging with unlimited penetration depth was employed to evaluate bio-circulation and distribution of Er-DCNPs-Dye-BP in healthy mice [41]. We utilized the phosphate groups on the surface of nanoparticles to label ${ }^{99 \mathrm{~m}} \mathrm{Tc}$ for tracing the circulation and distribution of nanoparticles (Fig. 4a). Time-dependent SPECT-CT images and the corresponding statistical analysis of major organs demonstrate that the accumulation of our nanoprobes in liver is over $20 \% \mathrm{ID} / \mathrm{g}$ and half-lifetime of blood circulation is $5.1 \pm 0.3 \mathrm{~h}$ (Figs. 4b-d and S17). To further demonstrate the biosafety of our nanoprobes, we performed hematoxylin-eosin (H\&E) staining of major organs (heart, liver, spleen, lung, kidney, brain) of mice. No clear pathological tissue is observed from mice in the experimental groups after different days post-injection of nanoprobes, in comparison with mice from the control group (Fig. 4e), which indicates an excellent biocompatibility of our nanoprobes. 

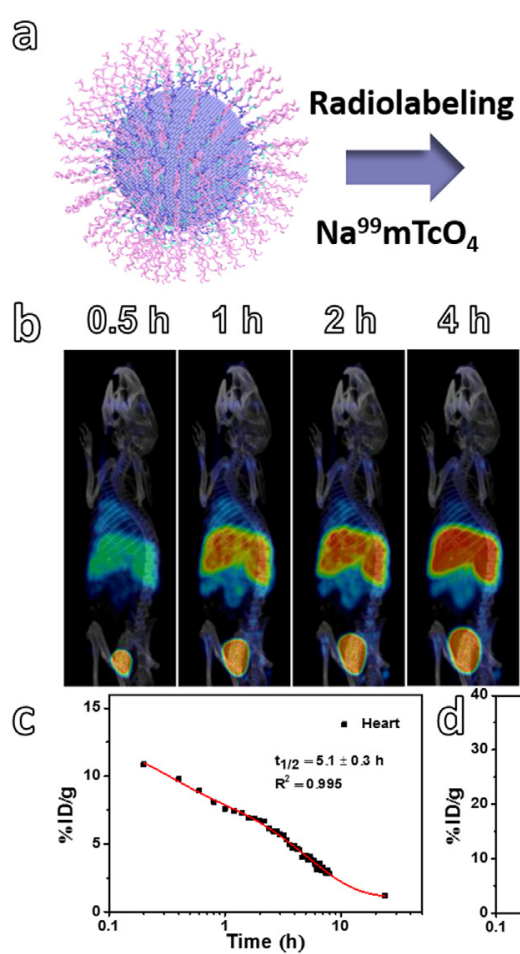

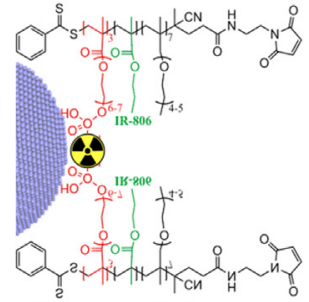

$4 \pi$ 6 解

8 ถ
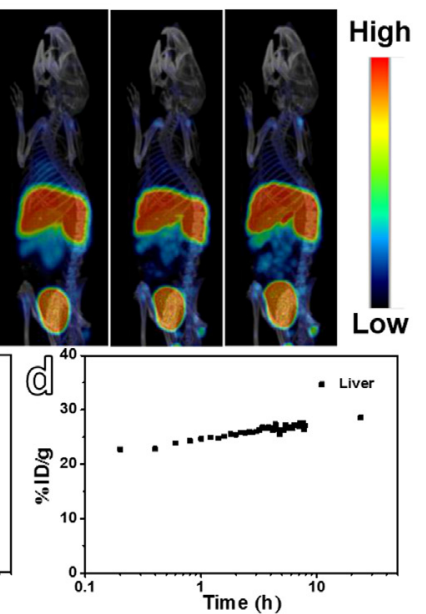

\section{(ㄷ)}

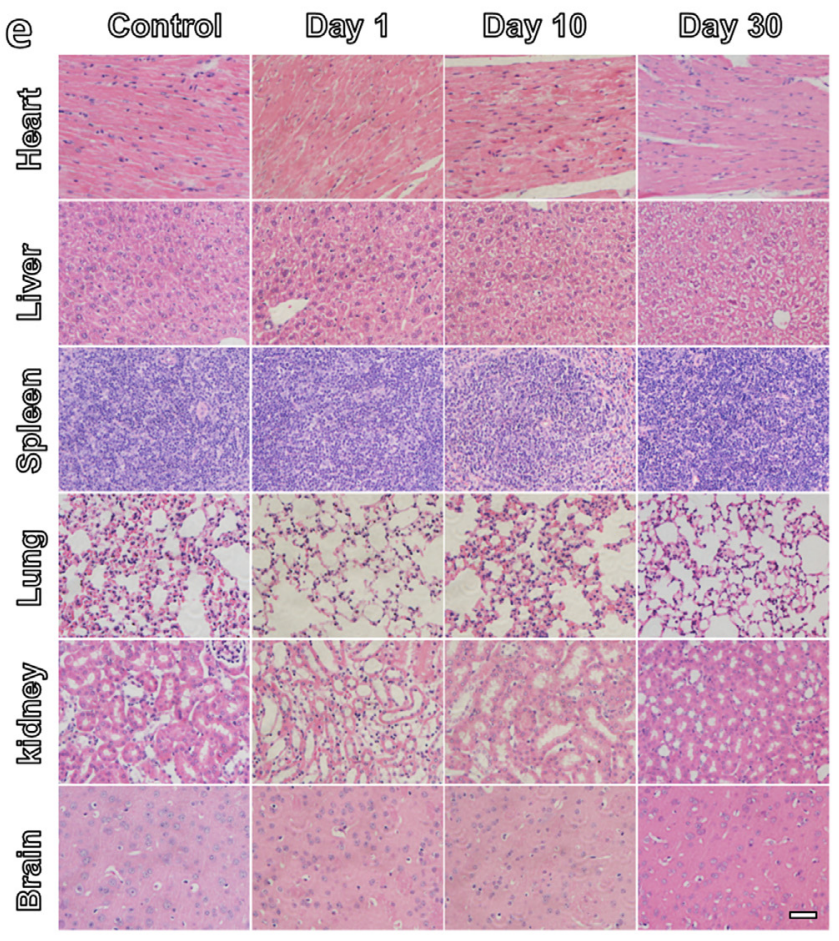

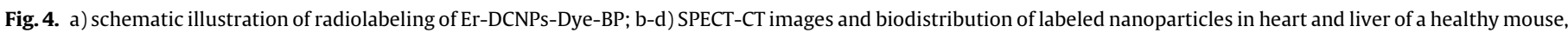

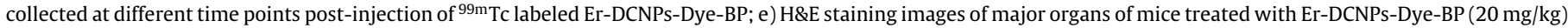
and sacrificed at different days in comparison with non-treated ones (control) (scale bar: $50 \mu \mathrm{m}$ ).

\section{NIR IIb fluorescence imaging of orthotopic glioma with nanoprobes}

Encouraged by the above experimental results, we evaluated the capability of our nanoprobes for actively targeted imaging of orthotopic gliomas. We first employed $T_{2}$-weighted MRI to locate deep-seated and small-sized orthotopic gliomas ( $\sim 3 \mathrm{~mm}$ in depth, $<3 \mathrm{~mm}$ in diameter) (Fig. S18a-left). Then, we performed NIR IIb fluorescence imaging of the orthotopic gliomas after injection of Er-DCNPs-Dye-BP and Er-DCNPs-Dye-BP-ANG nanoprobes, respectively. The brain vasculature is clearly observed in both cases, but the intratumoral enrichment of nanoprobes through the enhanced permeability and retention effect (EPR) alone is low due to the protection of BBB and non-specificity to tumor (Fig. S18a-right). In contrast, ANG functionalized nanoprobes can be efficiently accumulated in the tumor, leading to a higher tumor-to-background ratio (TBR) of 7.0 at 1 min post tail-vein injection, which is 1.8 fold that obtained from injection of Er-DCNPs-Dye-BP probes (Fig. $\mathrm{S} 18 \mathrm{~b})$.

To further improve the intratumoral accumulation of nanoprobes for the NIR IIb fluorescence imaging, we used focused ultrasound sonication (FUS) to assist the delivery of nanoprobes. Previous reports have demonstrated the advances of the FUS technique in controllable opening of BBB for passively delivering nanoprobes to glioma $[34,42,43]$. Since active targeting enables more accumulation of nanoprobes in the tumor [44], we believe that temporary opening of BBB by FUS could efficiently facilitate the intratumoral accumulation of nanoprobes for precisely delineating glioma margins and imaging-guided surgery. Under the guidance of pre-positioned $T_{2}$-weighted MRI and NIR IIb fluorescence imaging of cerebrovascular network (Fig. 5a), we could precisely pinpoint the position of the tumor and use the FUS to open the local BBB in conjugation with microbubbles. MRI images show that the established orthotopic gliomas were seated below the skull of mice by around $3.7 \mathrm{~mm}$ with a diameter smaller than $3 \mathrm{~mm}$ (Fig. 5b-left, white dashed line). Fluorescence imaging was then carried out at different time points after intravenous injection of NIR IIb nanoprobes (Fig. 5b-right). Under the assistance of FUS, much brighter tumors are observed than without FUS treatment. The TBR for the mice administered Er-DCNPs-Dye-BP-ANG could be up to 12.5 at 25 min post-FUS treatment (Fig. 5c), which is higher than that (10.2) of mice intravenously injected with Er-DCNPs-Dye-BP, due to the synergetic effects of FUS and active targeting. In addition, the TBR decline half-time for the group of mice administered Er-DCNPs-Dye-BP-ANG is around $150 \mathrm{~min}$, which is longer than that for mice injected with Er-DCNPs-Dye-BP (only $\sim 90 \mathrm{~min}$ ). The results clearly show that the absence of tumortargeting peptide resulted in a relatively low TBR and reduced the retention time of nanoparticles. Previously, we showed that active targeting strategy alone could not ensure and control sufficient nanoprobes to cross BBB and accumulate within the tumor. Since glioma has higher vascular density than normal brain tissue by over 3-fold (Fig. S19), FUS treatment could also produce ruptures in the vasculature to increase its permeability for nanoprobes diffusing into the tumor [45], which would further lead to strong NIR II fluorescence and a high TBR.

Similarly, the full width at half maximum (FWHM) of the intensity profile can be used to characterize the diameter of vessels or the size of tumors. The GaussAMP fitted FWHM of the vessel at the tumor spot is measured to be $0.45 \mathrm{~mm}$ in the image acquired at 1 min post-injection (Fig. 5d). More importantly, the sizes of tumors measured from the FWHM of the fluorescence intensity acquired at $25 \mathrm{~min}$ post-FUS treatment (Fig. $5 \mathrm{e}-\mathrm{f}$ ) are $2 \mathrm{~mm}$ and $3 \mathrm{~mm}$ (from multiplying the FWHM by 2) for the mice injected with Er-DCNPsDye-BP and Er-DCNPs-Dye-BP-ANG, respectively, which are very close to those (i.e., $1.7 \mathrm{~mm}$ and $2.7 \mathrm{~mm}$ respectively) obtained from $T_{2}$-weighted MRI images due to the low background of NIR IIb fluorescence. The subsequent blood vessel and rare-earth staining of tumor tissues confirm that lanthanide nanoprobes were still retained in the vasculature-abundant tumor tissue (black arrow), 


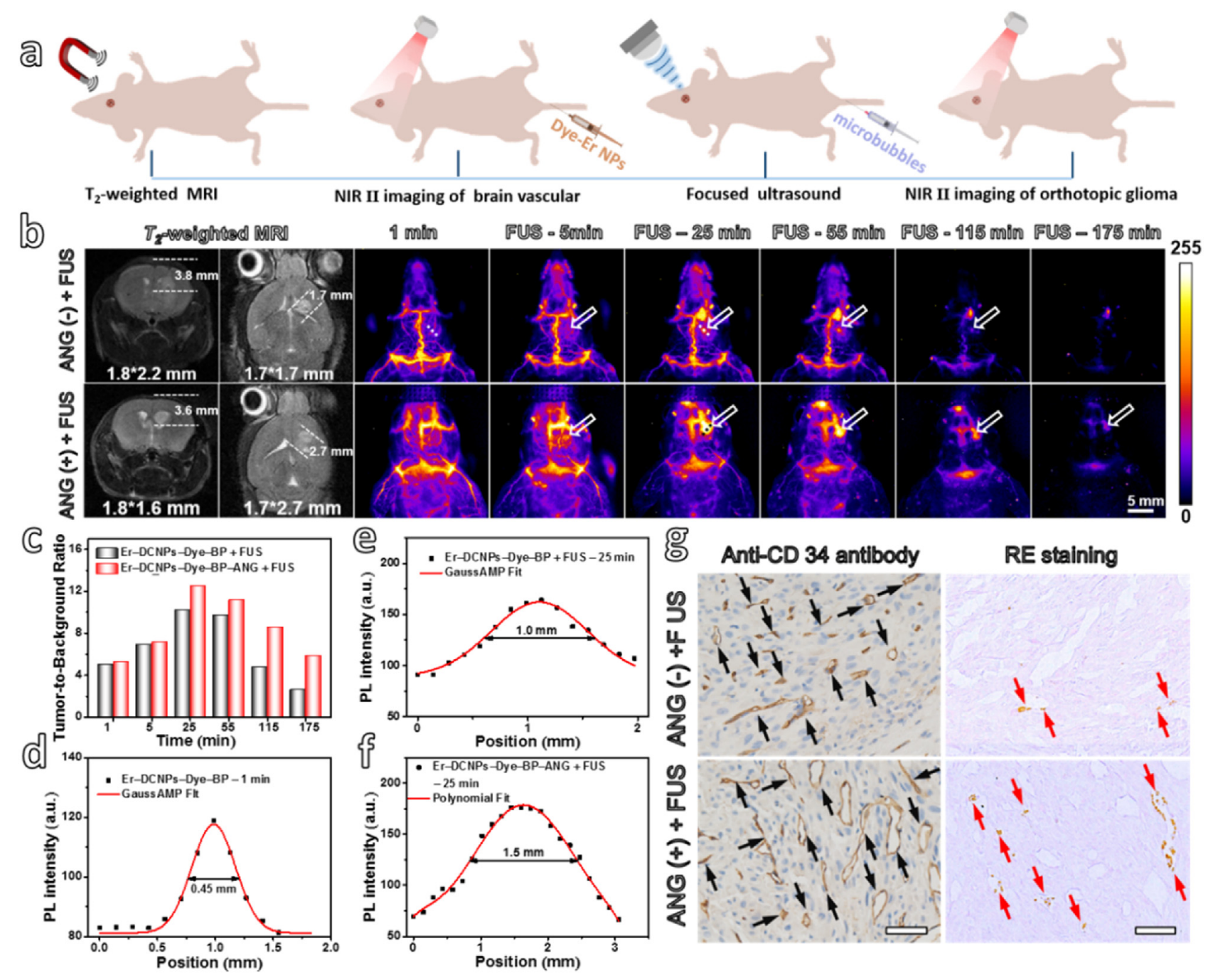

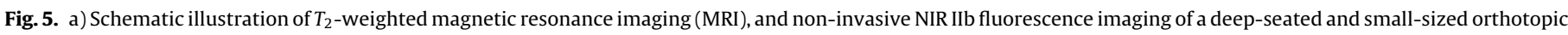

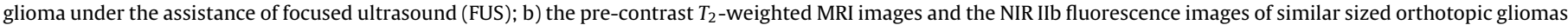

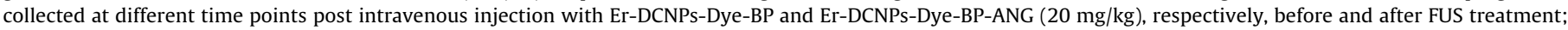

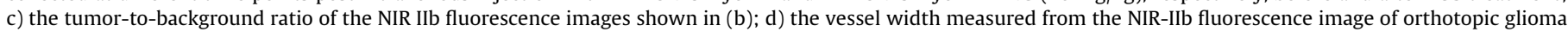

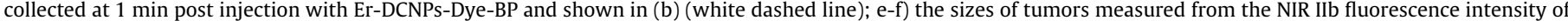

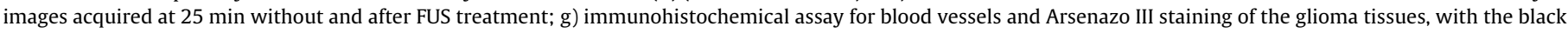
and red arrows indicating the capillaries and rare-earth ions in the tumor tissues (scale bar: $50 \mu \mathrm{m}$ ).

even 180 min after NIR IIb fluorescence imaging, and there was longer retention of Er-DCNPs-Dye-BP-ANG than Er-DCNPs-Dye$\mathrm{BP}$ in the tumor, as shown in Fig. 5g-right (red arrow). Based on these results, we can claim that sequential operations with prepositioned $T_{2}$-weighted MRI and preoperative NIR IIb fluorescence imaging enable us to pinpoint the location of deep-seated and small-sized orthotopic gliomas for precisely depicting the margins of tumors with a high TBR under the cooperation of active targeting and FUS treatment.

Surgical resection of orthotopic glioma guided by NIR IIb fluorescence imaging

The closely matching tumor size from NIR IIb fluorescence imaging and MRI suggests that portable NIR II fluorescence imaging could be used for imaging-guided surgery due to the significance of real-time visualization of tumor margins with precision. To clearly show the margin of tumor, we first used $T_{2}$-weighted MRI and NIR IIb fluorescence imaging to locate the glioma, applied the FUS treatment, and then did cardiac perfusion to eradicate the interference of nanoprobes in the blood. After that, we resected the orthotopic glioma under the guidance of NIR IIb fluorescence imaging (Fig. 6a). Large-sized gliomas were used to test the feasibility of this designed operational procedure. The $T_{2}$-weighted MRI images of brain show that the sizes of the tumors are $7.9 \mathrm{~mm}$ and $6.8 \mathrm{~mm}$ in diameter for the mice from the ANG(+) + FUS and ANG(-) + FUS groups, respectively, which are close to the size of the gliomas measured from the NIR IIb fluorescence images (e.g. $8.1 \mathrm{~mm}$ and $8.5 \mathrm{~mm}$, respectively, in Fig. 6b-d). The smaller difference in the tumor size between MRI and NIR IIb fluorescence images $(0.2 \mathrm{~mm})$ in the ANG(+) + FUS group further supports the more accumulation of nanoprobes within the tumor and strong NIR IIb fluorescence, due to the tumor-targeting capacity of the ANG peptide. In contrast, non-specific binding caused only a low accumulation of nanoprobes and weaker luminescence, resulting in a notable size difference (1.7 $\mathrm{mm})$.

To further address the great potential of NIR IIb fluorescence in imaging-guided surgery of orthotopic glioma, we utilized ErDCNPs-Dye-BP-ANG as probes to perform the surgical resection of small gliomas. Small gliomas $(3.8 \mathrm{~mm}$ in depth and $2 \mathrm{~mm}$ in 


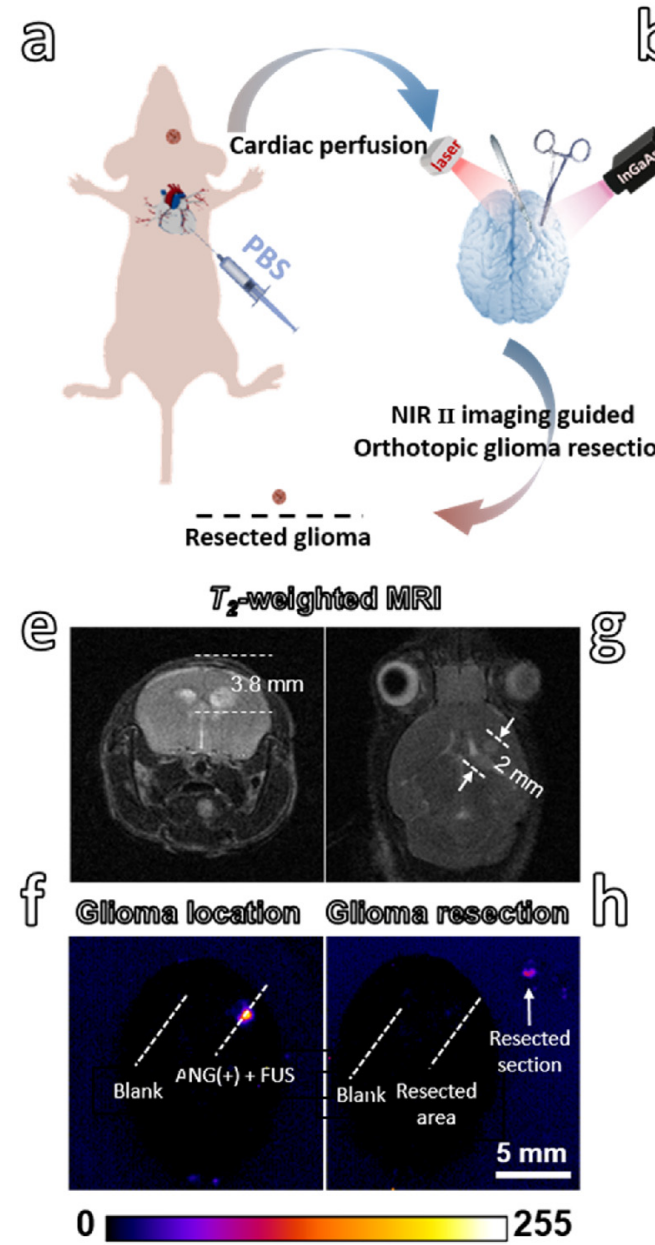

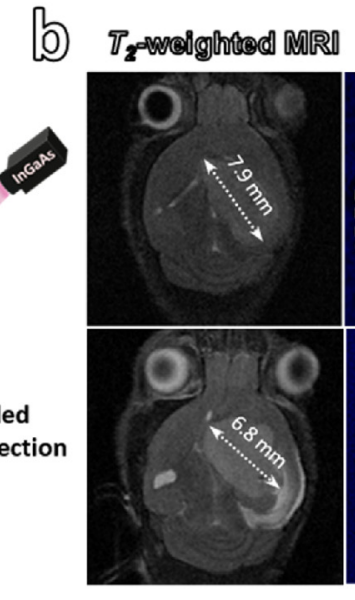
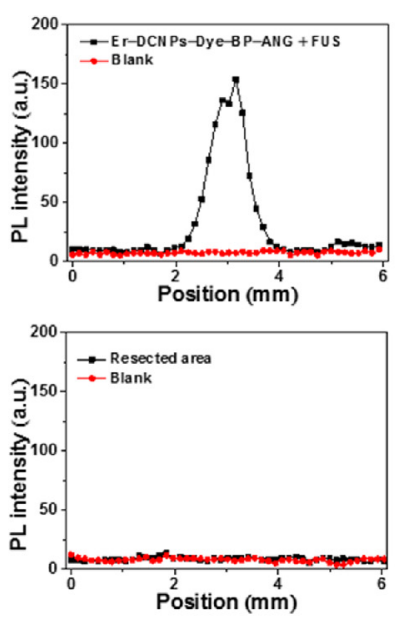

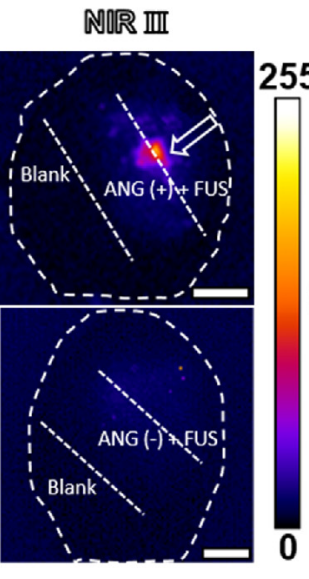

Ø̊
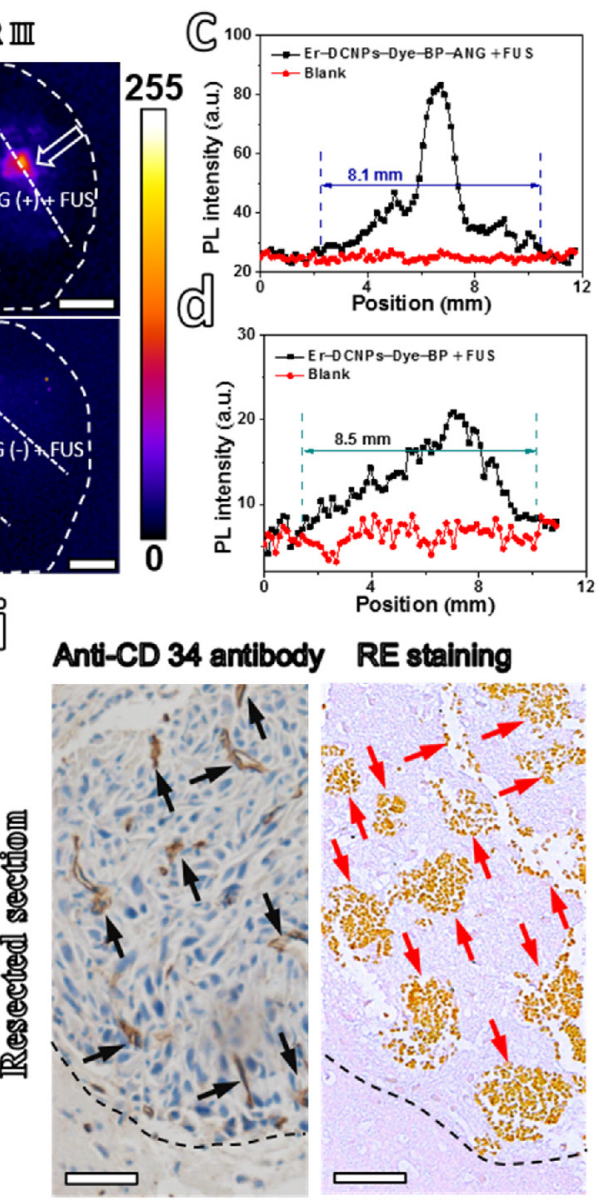

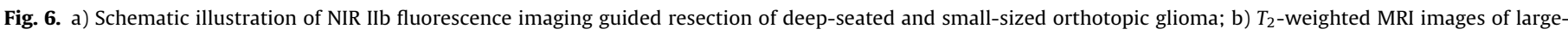

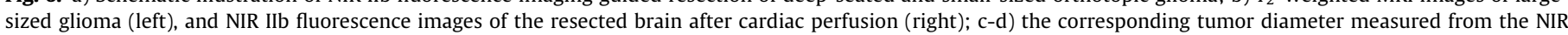

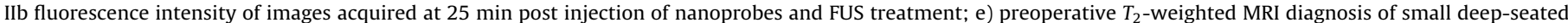

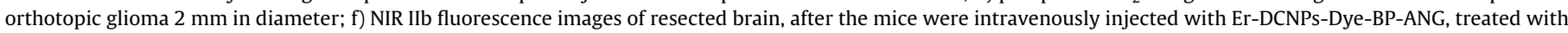

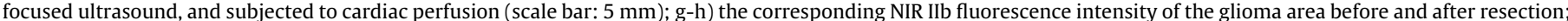

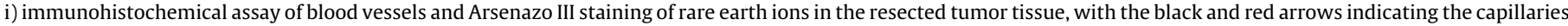
and rare-earth ions (scale bar: $50 \mu \mathrm{m}$ ).

diameter) were measured with $T_{2}$-weighted MRI (Fig. 6e). After the NIR IIb fluorescence imaging proceeded at 25 min post FUS treatment, similar cardiac perfusion and craniotomy were carried out to reduce the interference from blood. The TBR of the located glioma is up to 150 in comparison with normal brain tissue (blank), and the corresponding tumor size measured from NIR IIb fluorescence intensity is $2 \mathrm{~mm}$ in diameter (Figs. 6f-left, 6g), which is exactly same as for the $T_{2}$-weighted MRI images. After the resection of a glioma under the guidance of NIR IIb fluorescence imaging, no fluorescence signal was left in the surrounding tissue of the resected tumor compared to the normal brain tissue (Fig. 6f-right, 6h). The high TBR is mainly attributed to the high accumulation of ErDCNPs-Dye-BP-ANG (red arrow) in the tumor area with abundant vasculature (black arrow), which is confirmed through blood vessel and rare-earth staining analyses of the resected section (Fig. 6i). These results demonstrate that intraoperative NIR IIb fluorescence imaging is promising for finely delineating tumor margins and imaging-guided surgery.

\section{Conclusions}

In summary, we proposed an energy-cascaded downconversion strategy for drastically enhancing $1525 \mathrm{~nm}$ emission of Er-based DCNPs, and an efficient delivery of resultant highly bright nanoparticles for NIR IIb fluorescence imaging of brain tumors and imaging-guided surgery. Specifically, we designed energycascaded $\mathrm{NaErF}_{4} @ \mathrm{NaAF}_{4} @ \mathrm{NaLuF}_{4}(\mathrm{~A}=\mathrm{Yb}, \mathrm{Ho}, \mathrm{Tm}) \mathrm{DCNPs}$, in which three interlayers with different components and thickness were firstly investigated their energy tapping effects on DC process, secondly, $\mathrm{Ce}^{3+}$ ions were doped into $\mathrm{NaErF}_{4}$ core to maximize ECD process for improving ${ }^{4} \mathrm{I}_{13 / 2} \rightarrow{ }^{4} \mathrm{I}_{15 / 2}$ transition, and finally Dye-BP containing IR-806 as a major energy harvester was used to modify nanoparticle surface. The efficient energy cascade in the optimized nanoparticles (Er-DCNPs-Dye-BP) synergistically boosted $1525 \mathrm{~nm}$ fluorescence by 675 times in aqueous solution, in comparison with that of $\mathrm{NaErF}_{4}$ nanoparticles. We modified the Er-DCNPs-Dye-BP with tumor-targeting peptide and used the FUS technique to temporarily open the BBB to cooperatively deliver them into orthotopic glioma. Owing to the strong fluorescence of our nanoprobes, we can precisely delineate the tumor margin, and resect them under the guidance of NIR IIb fluorescence imaging with a high TBR. The sizes of the tumors measured from the intensity of NIR II fluorescence are very close to those measured from preoperative $T_{2}$-weighted MRI images, due to the low background of NIR IIb fluorescence from tissue. These results demonstrate the great potential of our strategies in engineering NIR IIb fluorescence of lanthanide nanoparticles and in imaging-guided surgery of tumor. The results also demonstrate the promise of efficient delivery of theranostic nanoparticles for 
diagnosis and treatment of tumor by combining active targeting strategy with focused ultrasound (FUS) technique.

\section{Materials and methods}

\section{Materials}

$\mathrm{ErCl}_{3} \cdot 6 \mathrm{H}_{2} \mathrm{O}, \mathrm{CeCl}_{3} \cdot 7 \mathrm{H}_{2} \mathrm{O}, \mathrm{YbCl}_{3} \cdot 6 \mathrm{H}_{2} \mathrm{O}, \mathrm{TmCl}_{3} \cdot 6 \mathrm{H}_{2} \mathrm{O}, \mathrm{HoCl}_{3} \cdot 6 \mathrm{H}_{2} \mathrm{O}$, $\mathrm{LuCl}_{3} \cdot 6 \mathrm{H}_{2} \mathrm{O}$, sodium fluoride (NaF, $99.99 \%$ ), oleic acid (OA, $85 \%$ ), 1-octadecene (ODE, $90 \%$ ), 4-mercaptobenzoic acid (90\%), 2-aminoethyl methacrylate hydrochloride (AMA, 90 $\%$ ), N,N'-dicyclohexylcarbodiimide (DCC, $99.0 \%$ ), 4-cyano4-(phenylcarbonothioylthio)pentanoic acid (CTA, >97\%), 2,2'-azobis(2-methylpropionitrile) (AIBN, $99 \%)$, and tris(2carboxyethyl)phosphine (TCEP, $98 \%$ ) were purchased from Aladdin Co. Ltd. Sodium hydroxide $(\mathrm{NaOH}, 96 \%)$ was purchased from Beijing Chemical Reagents Co. Ltd. Tris buffer solution $(\mathrm{pH}=$ 8.0) and physiological saline were obtained from Shanghai Yuanye Biotech, Inc. Thiolated-Angiopep-2 (TFFYGGSRGKRNNFKTEEYC, ANG-SH) was purchased from Xi'an Ruixi Biological Technology Co. Ltd. Poly(ethylene glycol)methyl ether methacrylate (number average molecular weight, $\mathrm{M}_{\mathrm{n}}=300$, containing 100 ppm hydroquinone monomethyl ether (MEHQ) and $300 \mathrm{ppm}$ butylated hydroxytoluene (BHT) as inhibitor), and Poly(ethylene glycol)methacrylate (average $M_{n}=360$, containing $500-800 \mathrm{ppm}$ MEHQ as inhibitor) were purchased from Sigma-Aldrich. N-(2aminoethyl)maleimide hydrochloride (HPLC, >93.0\%) was bought from TCI Co., Ltd. Phosphorus oxychloride (99\%) was purchased from Xiya Reagent. All reagents were used as received without any purification.

\section{Synthesis of Er-based core-shell nanoparticles}

Synthesis of $\mathrm{NaErF}_{4} @ \mathrm{NaAF}_{4}(x \mathrm{~nm}, \mathrm{~A}=\mathrm{Yb}, \mathrm{Ho}$, and $\mathrm{Tm}) @ \mathrm{NaLuF}_{4}$ core-shell-shell nanoparticles

The $\mathrm{NaLnF}_{4}(\mathrm{Ln}=\mathrm{Er}, \mathrm{Ce}, \mathrm{Yb}, \mathrm{Lu})$ precursors were prepared as described elsewhere $[30,46,47]$, and the resultant $\mathrm{NaLnF}_{4}$ nanoclusters were dispersed in cyclohexane for further use. $\mathrm{NaErF}_{4}$ nanoclusters solution ( $2 \mathrm{~mL}, 0.5 \mathrm{mmol}$ ) was mixed with $\mathrm{OA}(4 \mathrm{~mL})$ and ODE $(10 \mathrm{~mL})$ in a three-neck flask $(100 \mathrm{~mL})$. The flask was purged with nitrogen $\left(\mathrm{N}_{2}\right)$ at $70{ }^{\circ} \mathrm{C}$ for 30 min to fully remove the cyclohexane, and then heated up to $280^{\circ} \mathrm{C}$ at a rate of $\sim 10^{\circ} \mathrm{C} / \mathrm{min}$. After reacted at $280{ }^{\circ} \mathrm{C}$ for $30 \mathrm{~min}$, the solution was cooled to 70 ${ }^{\circ} \mathrm{C}$, followed by the addition of the mixture of $\mathrm{NaYbF}_{4}$ nanoclusters solution $(0.2,0.4,1 \mathrm{~mL}, 0.25 \mathrm{mmol} / \mathrm{mL}), \mathrm{OA}(2 \mathrm{~mL})$ and ODE $(5 \mathrm{~mL})$. The resultant solution was maintained at $70{ }^{\circ} \mathrm{C}$ for $20 \mathrm{~min}$, heated up to $280{ }^{\circ} \mathrm{C}$ at the rate of $\sim 10{ }^{\circ} \mathrm{C} / \mathrm{min}$, and then kept at $280{ }^{\circ} \mathrm{C}$ for 30 min to form $\mathrm{NaErF}_{4} @ \mathrm{NaYbF}_{4}$ nanoparticles with different thicknesses of their $\mathrm{NaYbF}_{4}$ shells. The growth of $\mathrm{NaLuF}_{4}$ inert shells is similar to the growth of $\mathrm{NaYbF}_{4}$ shells, except that the amount (2 $\mathrm{mL}, 0.25 \mathrm{mmol} / \mathrm{mL})$ of precursor and the reaction time at $280^{\circ} \mathrm{C}(60$ min) were fixed. After cooling to room temperature, the reaction mixture was centrifuged at $11,000 \mathrm{rpm}$ for $5 \mathrm{~min}$ to collected coreshell-shell nanoparticles, which were washed twice with absolute ethanol and finally dispersed in tetrahydrofuran (THF).

The synthesis and purification of $\mathrm{NaErF}_{4} @ \mathrm{NaHoF}_{4}(\mathrm{x} \mathrm{nm}, \mathrm{x}=0.2$, 0.8, 1.1, 1.8)@NaLuF 4 , and $\mathrm{NaErF}_{4} @ \mathrm{NaTmF}_{4}(\mathrm{x} \mathrm{nm}, \mathrm{x}=0.4,0.8,1.6$, 2.1)@NaLuF 4 core-shell-shell nanoparticles were same to the above methods, except the $\mathrm{NaHoF}_{4}$ and $\mathrm{NaTmF}_{4}$ nanoclusters and their amounts.

Synthesis of NaErF $: y \% \mathrm{Ce}(y=1,2.5,5,10) @ \mathrm{NaYbF}_{4}(0.9$

$n \mathrm{~m}) @ \mathrm{NaLuF}_{4}$ core-shell-shell nanoparticles

Doping different concentrations of $\mathrm{Ce}^{3+}$ ions into the host matrix of $\mathrm{NaErF}_{4}$ was carried out by using the Liquid-SolidSolution (LSS) strategy at room temperature. The growth method for $\mathrm{NaErF}_{4}: \mathrm{y} \% \mathrm{Ce}(\mathrm{y}=1,2.5,5,10)$ cores, and $\mathrm{NaYbF}_{4}$ and $\mathrm{NaLuF}_{4}$ shells was exactly same as that used above.

Modification of Er-DCNPs with Dye-BP and ANG-SH (simplified to Er-DCNPs-Dye-BP-ANG)

To optimize the dye sensitization, the oleic acid on the surface of Er-DCNPs was replaced with different ratios of Dye-BP through ligand exchange, as reported previously [37]. Typically, $10 \mathrm{mg}$ ErDCNPs were respectively mixed with BP and Dye-BP in a mass ratio of 50:0; $50: 10 ; 50: 30 ; 50: 50 \mathrm{mg}$ ), or mixed with Dye-BP alone (50, $100,200 \mathrm{mg})$ in THF $(5 \mathrm{~mL})$. The solution was stirred overnight. Then, the polymer coated Er-DCNPs were precipitated by adding cyclohexane and dried under a vacuum at room temperature for $2 \mathrm{~h}$. The dried nanoparticles were redispersed in deionized water $(5 \mathrm{~mL})$, and the resultant solution was purified by ultrafiltration three times with the centrifugal force of $1500 \mathrm{~g}$ to remove free polymer.

The synthesis of ANG-SH peptide functionalized Er-DCNPs-DyeBP was conducted referring to the typical "click reaction". ANG-SH peptide $(2 \mathrm{mg})$ and TCEP $(1 \mathrm{mg})$ were first dissolved in $3.5 \mathrm{~mL}$ of Tris buffered saline solution, followed by the addition of Er-DCNPs-DyeBP $(2 \mathrm{mg})$. After stirring for $1 \mathrm{~h}$ at room temperature, the reaction solution was purified three times by ultrafiltration with the centrifugal force of $1000 \mathrm{~g}$ times to remove the TCEP and free ANG-SH, and then the final Er-DCNPs-Dye-BP-ANG was obtained.

\section{Characterization}

TEM images were collected with a FEI Tecnai G20 transmission electron microscope operating with $200 \mathrm{kV}$ acceleration voltage. X-ray Energy Dispersive Spectroscopy (EDS) elemental mapping, high-resolution TEM and high angle annular dark field scanning transmission electron microscopy (HAADF-STEM) imaging (Zcontrast imaging mode) were performed on a JEOL-ARM200 Cold FEG transmission electron microscope operating at $80 \mathrm{kV}$, equipped with a double spherical aberration correctors and fitted with a JEOL SDD CENTURIO EDS system. The fluorescence spectra were recorded on a FLS980 spectrometer (Edinburgh Instruments, UK) equipped with excitation sources ( $808 \mathrm{~nm}$ and $980 \mathrm{~nm}$ continuouswave laser diodes). The hydrodynamic size was measured at $25^{\circ} \mathrm{C}$ with a Malvern Zetasizer Nano ZS90 equipped with a solid state He-Ne laser $(\lambda=633 \mathrm{~nm})$.

\section{Animal tumor model}

All mice were cultured and experimented on in accordance with guidelines approved by the ethics committee of Soochow University (Soochow, China). Specific pathogen free (SPF) grade BALB/c nude mice (6-8 weeks old) were provided by the laboratory of the Animal Center of Soochow University. The xenograft tumor models were established by subcutaneous injection of U87 cells $\left(2 \times 10^{6}\right.$ in $50 \mu \mathrm{L}$ PBS) into the right flank region on the backs of the mice. The tumor imaging was carried out at 7-10 days after the inoculation of tumor cells. The orthotopic tumor models were established with the injection of $U 87$ cells $\left(5 \times 10^{5}\right.$ in $5 \mu \mathrm{L}$ PBS) into the striatum of mice. The injection location was set with the assistance of stereotaxic apparatus, which was bregma $1.0 \mathrm{~mm}$, right lateral $2.0 \mathrm{~mm}$, depth $2.5 \mathrm{~mm}$. To observe the growth of tumors, an MRS 3000 scanner ( $3 \mathrm{~T}$ ) was used to depict the size and depth of orthotopic gliomas by $T_{2}$-weighted imaging. The orthotopic glioma bearing mice were experimented on after different numbers of days $(7,14)$ after the inoculation of tumor cells. 


\section{Cytotoxicity assay}

The standard tetrazolium dye (MTT) assay was carried out to evaluate the cytotoxicity of Er-DCNPs-Dye-BP and Er-DCNPs-DyeBP-ANG towards U87 cancer cells. U87 cells $\left(1 \times 10^{4}\right.$ cells per well) were first seeded into 96-well plates and cultured for 24 $\mathrm{h}$ in a standard cell medium at $37{ }^{\circ} \mathrm{C}$ in $5 \% \mathrm{CO}_{2}$ atmosphere. Then, these cells were washed with phosphate buffered saline (PBS) and incubated with Er-DCNPs-Dye-BP and Er-DCNPs-DyeBP-ANG in different concentrations (i.e. 6.25, 12.5, 25, 50, 100, 200,400 , and $800 \mu \mathrm{g} / \mathrm{mL}$ ) for $24 \mathrm{~h}$. After that, the U87 cells were washed with PBS solution, followed by the addition of MTT [(3-(4,5dimethylthiazol-2-yl)-2,5-diphenyltetrazolium bromide)] solution $(20 \mu \mathrm{L}, 5 \mathrm{mg} / \mathrm{mL})$. After the cells were further incubated for $4 \mathrm{~h}$ and the culture medium was removed, $150 \mu \mathrm{L}$ dimethyl sulfoxide (DMSO) was added into each well. The absorbance of each solution at $490 \mathrm{~nm}$ was measured by a microplate reader (Thermo, Varioskan Flash).

\section{Cellular uptake}

U87 cells were seeded at a density of $2 \times 10^{4}-3 \times 10^{4}$ cells per cell culture dish and incubated for $24 \mathrm{~h}$ at $37^{\circ} \mathrm{C}$ under $5 \% \mathrm{CO}_{2}$ atmosphere. The Er-DCNPs-Dye-BP and Er-DCNPs-Dye-BP-ANG were dispersed into Dulbecco's Modified Eagle Medium (DMEM) for cellculture with a concentration of $400 \mu \mathrm{g} / \mathrm{mL}$, and then added into the culture dish after removal of the original culture medium. After co-incubation for $24 \mathrm{~h}$, the cells were washed two times with PBS to remove the non-uptake nanoparticles, and then fixed with $4 \%$ paraformaldehyde for $30 \mathrm{~min}$. The cells were washed twice with PBS solution, followed by the addition of $5 \mu \mathrm{g} / \mathrm{mL}$ Hoechst stain for staining the nuclei staining for $10 \mathrm{~min}$. The confocal fluorescence imaging experiments were carried out after washing three times with PBS.

\section{SPECT-CT imaging}

$2.5 \mathrm{mCi}{ }^{99 m} \mathrm{Tc}$ radionuclides (provided by Shanghai GMS Pharmaceutical Co., Ltd.) were used to label the Er-DCNPs-Dye-BP for SPECT-CT imaging. $200 \mu \mathrm{L}(2 \mathrm{mg} / \mathrm{mL})$ Er-DCNPs-Dye-BP solution was added into a freshly prepared stannous chloride $\left(\mathrm{SnCl}_{2}, 1\right.$ $\mathrm{mg} / \mathrm{mL}$ in $0.1 \mathrm{M} \mathrm{HCl}$ ) solution containing $2.5 \mathrm{mCi}{ }^{99 \mathrm{~m}} \mathrm{Tc}$ radionuclides and reacted for 30 min under ambient conditions. The mixed solution was ultra-filtrated for three times to remove free ${ }^{99 \mathrm{~m}} \mathrm{Tc}$ radionuclides using $30 \mathrm{kDa}$ Millipore membrane, and final labelling yield was $82.2 \%$. The resulting Er-DCNPs-Dye-BP-99m Tc solution was injected into an anaesthetized $B A L B / C$ healthy mouse for SPECT-CT imaging, which was recorded with the MILabs imaging system.

\section{Focused ultrasound sonication}

An ultrasound transducer $(0.5 \mathrm{MHz}$ in frequency and $30 \mathrm{~mm}$ in diameter) driven by a function generator and connected to a power amplifier was used to open the BBB of mice bearing orthotopic gliomas. The acoustic parameters were set at $0.5 \mathrm{MPa}$ in acoustic pressure, $0.5 \mathrm{MHz}$ in frequency, $1 \mathrm{~ms}$ in pulse interval, and $90 \mathrm{~s}$ for sonication. $50 \mu \mathrm{L}$ of microbubble solution $\left(\sim 1 \times 10^{9}\right.$ bubbles $\left./ \mathrm{mL}\right)$ was intravenously injected into the mice before ultrasound sonication.

\section{In-vivo NIR II fluorescence imaging}

In-vivo NIR II fluorescence imaging was conducted with a NIR II Imaging System (Serious II 900-1700, Suzhou NIR-Optics Co., Ltd.). The power density of the $808 \mathrm{~nm}$ laser was set to $100 \mathrm{~mW} / \mathrm{cm}^{2}$ for the subcutaneous glioma tumor model and $120 \mathrm{~mW} / \mathrm{cm}^{2}$ for the orthotopic glioma tumor imaging and $200 \mathrm{~mW} / \mathrm{cm}^{2}$ for imagingguided surgery. The mice were anaesthetized with isoflurane, placed in an animal bed, and then intravenously injected with ErDCNPs-Dye-BP and Er-DCNPs-Dye-BP-ANG in physiological saline solution with a dose of $12 \mathrm{mg} / \mathrm{kg}$ for the subcutaneous glioma tumor model, and $20 \mathrm{mg} / \mathrm{kg}$ for the orthotopic glioma tumor model, respectively. The NIR II fluorescence images were collected at different time points post injection. For the mice treated with FUS, their NIR II fluorescence images were collected at 1 min post injection of nanoprobes.

\section{Author statement}

F. R. performed the preparation, modification, and characterization of nanoprobes, and drafted the manuscript. H. L., H. Z., and Z. J. performed the cell and animal experiments. B. X. and T. H. synthesized and characterized the block polymers. C. G. and M. A. performed the TEM analysis of samples. Z. L., Q. S., and M. G. did the critical revisions of the manuscript.

\section{Declaration of Competing Interest}

The authors declare no conflict of interest.

\section{Acknowledgements}

Z. Li acknowledges support from the National Natural Science Foundation of China (81971671, 81527901), National Key Research and Development Program of China (2018YFA0208800), Jiangsu Provincial Key Research and Development Program (BE2019660). The authors also are grateful for support from the Jiangsu Provincial Key Laboratory of Radiation Medicine and Protection, the Priority Academic Development Program of Jiangsu Higher Education Institutions (PAPD). The authors would like to thank Dr. Tania Silver for helpful discussion of the manuscript. This project is co-funded by the European Union, the Région - Centre Val de Loire and The French minister of research (MESRI - DRRT). Europe is committed to the Centre-Val de Loire region with the European regional development fund (ERDF).

\section{Appendix A. Supplementary data}

Supplementary material related to this article can be found, in the online version, at doi:https://doi.org/10.1016/j.nantod.2020. 100905.

\section{References}

[1] J.Y. Lee, J.P. Thawani, J. Pierce, R. Zeh, M. Martinez-Lage, M. Chanin, O. Venegas, S. Nims, K. Learned, J. Keating, S. Singhal, Neurosurgery 79 (2016) 856-871.

[2] C. Li, L. Cao, Y. Zhang, P. Yi, M. Wang, B. Tan, Z. Deng, D. Wu, Q. Wang, Small 11 (2015) 4517-4525

[3] D.Y. Zhang, S. Singhal, J.Y.K. Lee, Neurosurgery 85 (2019) 312-324.

[4] P.L. Kubben, K.J. ter Meulen, O.E. Schijns, M.P. ter Laak-Poort, J.J. van Overbeeke, H. van Santbrink, Lancet Oncol. 12 (2011) 1062-1070.

[5] M.F. Kircher, J.K. Willmann, Radiology 264 (2012) 349-368.

[6] M.M. Kim, A. Parolia, M.P. Dunphy, S. Venneti, Nat. Rev. Clin. Oncol. 13 (2016) $725-739$.

[7] W. Stummer, U. Pichlmeier, T. Meinel, O.D. Wiestler, F. Zanella, R. Hans-Jurgen, Lancet Oncol. 7 (2006) 392-401.

[8] P. Schucht, K. Seidel, J. Beck, M. Murek, A. Jilch, R. Wiest, C. Fung, A. Raabe, Neurosurg. Focus 37 (2014) E16.

[9] J.X. Jiang, J.J. Keating, E.M. Jesus, R.P. Judy, B. Madajewski, O. Venegas, O.T Okusanya, S. Singhal, Am. J. Nucl. Med. Mol. Imaging 5 (2015) 390-400.

[10] R. Zeh, S. Sheikh, L. Xia, J. Pierce, A. Newton, J. Predina, S. Cho, M. Nasrallah, S. Singhal, J. Dorsey, J.Y.K. Lee,PLoS One 12 (2017), e0182034.

[11] W. Stummer, S. Stocker, S. Wagner, H. Stepp, C. Fritsch, C. Goetz, A.E. Goetz, R. Kiefmann, H.J. Reulen, Neurosurgery 42 (1998) 518-526.

[12] C. Li, Q. Wang, ACS Nano 12 (2018) 9654-9659. 
[13] Y. Xu, F. Ren, H. Liu, H. Zhang, Y. Han, Z. Liu, W. Wang, Q. Sun, C. Zhao, Z. Li, ACS Appl. Mater. Interfaces 11 (2019) 21399-21407.

[14] A.N. Bashkatov, E.A. Genina, V.I. Kochubey, V.V. Tuchin, J. Phys. D Appl. Phys. 38 (2005) 2543-2555

[15] G.S. Hong, A.L. Antaris, H.J. Dai, Nat. Biomed. Eng. 1 (2017) 0010.

[16] S. Diao, J.L. Blackburn, G. Hong, A.L. Antaris, J. Chang, J.Z. Wu, B. Zhang, K. Cheng, C.J. Kuo, H. Dai, Angew. Chem. Int. Ed. 54 (2015) 14758-14762.

[17] M.X. Zhang, J.Y. Yue, R. Cui, Z.R. Ma, H. Wan, F.F. Wang, S.J. Zhu, Y. Zhou, Y. Kuang, Y.T. Zhong, D.W. Pang, H.J. Daia, Proc. Natl. Acad. Sci. U. S. A. 115 (2018) 6590-6595.

[18] C. Sun, B. Li, M. Zhao, S. Wang, Z. Lei, L. Lu, H. Zhang, L. Feng, C. Dou, D. Yin, H. Xu, Y. Cheng, F. Zhang, J. Am. Chem. Soc. 141 (2019) 19221-19225.

[19] Y. Li, S. Zeng, J. Hao, ACS Nano 13 (2019) 248-259.

[20] Y. Zhong, Z. Ma, S. Zhu, J. Yue, M. Zhang, A.L. Antaris, J. Yuan, R. Cui, H. Wan, Y. Zhou, W. Wang, N.F. Huang, J. Luo, Z. Hu, H. Dai, Nat. Commun. 8 (2017) 737.

[21] L. Liu, S. Wang, B. Zhao, P. Pei, Y. Fan, X. Li, F. Zhang, Angew. Chem. Int. Ed. 57 (2018) 7518-7522.

[22] N.J. Johnson, S. He, S. Diao, E.M. Chan, H. Dai, A. Almutairi, J. Am. Chem. Soc. 139 (2017) 3275-3282.

[23] Y. Zhong, Z. Ma, F. Wang, X. Wang, Y. Yang, Y. Liu, X. Zhao, J. Li, H. Du, M. Zhang, Q. Cui, S. Zhu, Q. Sun, H. Wan, Y. Tian, Q. Liu, W. Wang, K.C. Garcia, H. Dai, Nat. Biotechnol. 37 (2019) 1322-1331.

[24] Y. Fan, L. Liu, F. Zhang, Nano Today 25 (2019) 68-84.

[25] Y.F. Wang, G.Y. Liu, L.D. Sun, J.W. Xiao, J.C. Zhou, C.H. Yan, ACS Nano 7 (2013) $7200-7206$.

[26] R. Wang, X. Li, L. Zhou, F. Zhang, Angew. Chem. Int. Ed. 53 (2014) 12086-12090

[27] C. Siefe, R.D. Mehlenbacher, C.S. Peng, Y. Zhang, S. Fischer, A. Lay, C.A. McLellan, A.P. Alivisatos, S. Chu, J.A. Dionne, J. Am. Chem. Soc. 141 (2019) 16997-17005

[28] Q. Chen, X. Xie, B. Huang, L. Liang, S. Han, Z. Yi, Y. Wang, Y. Li, D. Fan, L. Huang, X. Liu, Angew. Chem. Int. Ed. 56 (2017) 7605-7609.

[29] Y.F. Shang, S.W. Hao, W.Q. Lv, T. Chen, L. Tian, Z.T. Lei, C.H. Yang, J. Mater. Chem. C 6 (2018) 3869-3875.
[30] F. Ren, L. Ding, H. Liu, Q. Huang, H. Zhang, L. Zhang, J. Zeng, Q. Sun, Z. Li, M Gao, Biomaterials 175 (2018) 30-43.

[31] Y. Li, P. Zhang, H. Ning, J. Zeng, Y. Hou, L. Jing, C. Liu, M. Gao,Small 15 (2019), 1905344

[32] W. Wei, Y. Zhang, R. Chen, J. Goggi, N. Ren, L. Huang, K.K. Bhakoo, H. Sun, T.T.Y. Tan, Chem. Mater. 26 (2014) 5183-5186.

[33] B. Huang, Y. Zhou, P. Cheng, Z. Zhou, J. Li, G. Yang, J. Alloys. Compd. 686 (2016) 785-792.

[34] Z. Liu, F. Ren, H. Zhang, Q. Yuan, Z. Jiang, H. Liu, Q. Sun, Z. Li,Biomaterials 219 (2019), 119364.

[35] W. Shao, G. Chen, A. Kuzmin, H.L. Kutscher, A. Pliss, T.Y. Ohulchanskyy, P.N. Prasad, J. Am. Chem. Soc. 138 (2016) 16192-16195.

[36] G. Chen, J. Damasco, H. Qiu, W. Shao, T.Y. Ohulchanskyy, R.R. Valiev, X. Wu, G. Han, Y. Wang, C. Yang, H. Agren, P.N. Prasad, Nano Lett. 15 (2015) 7400-7407.

[37] C.Y. Liu, Z.Y. Gao, J.F. Zeng, Y. Hou, F. Fang, Y.L. Li, R.R. Qiao, L. Shen, H. Lei, W.S. Yang, M.Y. Gao, ACS Nano 7 (2013) 7227-7240.

[38] D. Ni, J. Zhang, W. Bu, H. Xing, F. Han, Q. Xiao, Z. Yao, F. Chen, Q. He, J. Liu, S. Zhang, W. Fan, L. Zhou, W. Peng, J. Shi, ACS Nano 8 (2014) 1231-1242.

[39] D. Furtado, M. Bjornmalm, S. Ayton, A.I. Bush, K. Kempe, F. Caruso,Adv. Mater. 30 (2018), e1801362.

[40] M. Zheng, Y. Liu, Y. Wang, D. Zhang, Y. Zou, W. Ruan, J. Yin, W. Tao, J.B. Park, B. Shi,Adv. Mater. 31 (2019), e1903277.

[41] R. Weissleder, M.J. Pittet, Nature 452 (2008) 580-589.

[42] H. Zhang, T. Wang, W. Qiu, Y. Han, Q. Sun, J. Zeng, F. Yan, H. Zheng, Z. Li, M. Gao, Nano Lett. 18 (2018) 4985-4992.

[43] H. Zhang, T. Wang, H. Liu, F. Ren, W. Qiu, Q. Sun, F. Yan, H. Zheng, Z. Li, M. Gao, Nanoscale 11 (2019) 7600-7608.

[44] M. Zhao, B. Li, Y. Fan, F. Zhang,Adv. Healthcare Mater. 8 (2019), e1801650.

[45] C.H. Fan, C.Y. Lin, H.L. Liu, C.K. Yeh, J. Control. Release 261 (2017) 246-262.

[46] L. Ding, F. Ren, Z. Liu, Z. Jiang, B. Yun, Q. Sun, Z. Li, Bioconjugate Chem. 31 (2020) 340-351.

[47] X. Wang, J. Zhuang, Q. Peng, Y. Li, Nature 437 (2005) 121-124. 